\title{
A POSSIBLE IDENTIFICATION OF THE QUASI-PERIODIC OSCILLATIONS IN DWARF NOVAE
}

\author{
D.E. Winget, \\ and \\ F. Wesemael, \\ and \\ H.M. Van Horn \\ Department of Physics and Astronomy \\ and \\ C.E. Kenneth Mees Observatory \\ Unfversity of Rochester
}

Quasi-periodic oscillations on timescales from $230 \mathrm{sec}$ to $150 \mathrm{~s}$ have been observed in several dwarf novae during outburst. These oscillations have been qualitatively interpreted as pulsations of the accretion disks in these systems. We explore this suggestion quantitatively by examining the non-radial pulsation properties of viscous accretion disks in the linear, quasi-adiabatic approximation. The weak coupling of the pulsation modes in the radial ( $\bar{w}-)$ direction is consistent with the interpretation of these modes as arising in Individual disk annuli with periods and elgenfunctions characteristic of the properties of the annulus. The periods of the oscillations, which are periodic in $\phi, z$, and $t$, are shown to be directly proportional to the Keplerian period of the annulus. The existence of a luminosity maximum at some radial position in the disk suggests that the annull near this position w111 be the dominant contributors to the observed oscillations. For parameters typical of dwarf novae, the corresponding oscillation periods are $\sim 20 \mathrm{~s}$, with a range from $\sim 10 \mathrm{~s}$ to $\sim 150 \mathrm{~s}$. We suggest that these modes correspond to the observed quasi-perfodic oscillations. 